- Reduced pressure on nursing staff with more nurses sharing the burden of medicines rounds with round size reducing from $7 / 8$ patients to 5 patients

- Patients receiving their medicines in a more timely fashion; staff able to spend more time on clinical care

- 'Woody' is a valuable reminder to staff to return to patients if necessary

- Controlled Drug documentation is checked and completed daily.

Conclusion Focusing on policy implementation and revising medicines training results in a measurable reduction in documentation errors. However, involving a wider team in developing simple, practical ideas leads to improved medicines administration for patients; reduced pressure on nurses; and better staff morale.

Small changes really can lead to big improvements.

\section{P-132 DEMENTIA IN A HOSPICE? WHERE DO WE START?}

1,2Sharron Tolman. 'St Cuthbert's Hospice, Durham, UK; ${ }^{2}$ Dementia UK

\subsection{6/bmjspcare-2016-001245.155}

I joined St Cuthbert's Hospice as an Admiral Nurse in 2014. Many hospices have contacted us since to enquire about our work so I thought this would be a good opportunity to share what we have achieved so far.

We began with challenging the fear and stigma of dementia by raising awareness among all staff, from all departments, some becoming dementia friends and champions. A gentleman with dementia helped us with improved signage to reduce confusion and aid orientation throughout the building. We were talking about dementia more, seeing the person first, and bringing it into mandatory training for staff and volunteers. We built on compassion and confidence to care, embedding assessments which would impact on improved care outcomes and safety-specific tools for cognitive testing, pain, delirium and depression. We helped staff understand the difference between the 3 Ds - delirium, depression and dementia - and the importance of knowing this to provide the most appropriate intervention. We developed a delirium leaflet for patients and carers to help people understand what is happening to them when they are confused and how carers can help.

At the same time, we engaged with the community and all the other dementia care providers. Positive, collaborative relationships emerged, encouraging referrals and an understanding of what else is out there. If the hospice can't help, who can? We provide telephone, website and face to face contact for carers of people with dementia, at all stages of the illness but more commonly in the moderate to late stages as things are changing and carers are hitting crisis points. We are developing a volunteer-led project, providing companionship to those with advanced dementia at home, looking at new ways of communicating as verbal skills are lost and we have plans for cognitive stimulation groups for people with mild to moderate dementia. Exciting times!

\section{P-133 HOSPICE ENABLED DEMENTIA CARE: EXPLORING A PATIENT AND CARER LED COLLABORATIVE HUB RESOURCE}

Maddy Bass, John Hunt. St John's Hospice, Lancaster, UK

10.1136/bmjspcare-2016-001245.156
'Hospice enabled dementia care: [hospices as] key partners working with their local community and care economy to ensure people affected with dementia have access to optimum palliative care and support wherever they live and wherever they need it, regardless off anticipated prognosis'.

St John's Hospice is committed to reaching out to people affected by dementia and is developing a strategic action plan and partnerships to best support individuals with a dementia diagnosis and those closest to them.

In order that the most effective assistance, information and care is made available, the hospice, together with NHS, social care, legal and university researcher colleagues has adopted a pilot model developed by a voluntary group, Lancashire Dementia Voices (LDV) within the region. In March 2016, this independent group of people living with dementia, launched a project to provide a 'one-stop shop' resource, regularly monthly and with themes and speakers to ensure up-to-date information, advice and support is readily available.

St John's Hospice and its partners see this 'Hub' resource as a potentially creative and collaborative development. Its first action is to seek the views of people living with dementia, through a 'listening event' to be held at the hospice in early June 2016. Information will be disseminated directly to individuals and their carers, identified through NHS services. It is also hoped that this event will generate interest for volunteers from the target group to join the multiple agencies in a steering group to determine the structure, venue, initial programme and regularity of the 'Hub' service.

The hospice views this as part of its increasing community engagement and will review and evaluate its contribution to the project. By absorbing the views of people affected by dementia, and their carers, the hospice hopes to ensure delivery of dementia-friendly and effective inpatient, outpatient and home services.

\section{P-134 HOSPICE AND DEMENTIA CARE: INNOVATION AND COLLABORATION}

Heather Watson. Dorothy House Hospice Care, Bradford on Avon, UK

\subsection{6/bmjspcare-2016-001245.157}

Background While few people challenge the belief that dementia is a life-limiting illness, it has struggled to be accorded the same degree of service provision within the palliative care domain as other life-limiting illnesses (Hospice UK, 2015). There are differing reasons for this ranging from the historical focus of hospice care which has traditionally not included individuals with a dementia diagnosis, to concerns about knowledge and skills of staff, and resource implications of an extending service.

Key Issues Referrals to the hospice for people with dementia are generally low. Anecdotal evidence from discussions with local dementia services highlighted that many staff are aware of the role of hospice care in dementia. The Hospice Dementia Working Group therefore reviewed its provision of end of life care for people with dementia identifying areas for development mapped against the Southwest Hospital Standards in Dementia Care.

Method Following the review a dementia strategy was developed with six key actions for the next year: To develop a Dementia friendly environment; Workforce dementia awareness training for all staff; The rollout of a training programme for dementia care professionals to support end-of-life care locally, raising awareness of the role of hospice care; Representation at local dementia strategy groups to contribute an end of life perspective 
to dementia service plans; To build collaborative and mutually supportive ways of working with local dementia services; To engage with the community in the development of Dementia Action Alliances.

Conclusions Key learning from this process is that hospices need to be proactive to ensure their service is responsive to people with dementia. Community engagement and cross organisational working is crucial to this with the Hospice being involved in dementia service development and highlighting their commitment to equality of access for people with dementia and their families.

\section{P-135 THE HOSPICE DEMENTIA NURSE FOR CARE HOMES PROJECT}

Janet Willoughby. The Hospice of St Francis, Berkhamsted, UK

\subsection{6/bmjspcare-2016-001245.158}

Context People with dementia may receive sub-optimal end-oflife care (EoLC) in comparison with those who are cognitively intact (Lillyman and Bruce, 2016). Care home staff report feeling poorly-equipped to manage EoLC in dementia (Kupeli et al., 2016). Anecdotal evidence from the delivery of the ABC End of Life Care education programme for care homes by the Hospice of St Francis found challenges for staff included assessment, recognition of dying and symptom control.

The Dementia Nurse in Care Homes project aims to support care home staff in improving the quality of life of people with dementia and their families. A dementia nurse from the Hospice of St Francis will work in three specialist dementia units, a residential care home, a nursing home and a mental health care unit. Objectives

1. Provide clinical support to care home staff to recognise when the person is dying.

2. Promote compassionate, holistic care in the individual's familiar environment, preventing inappropriate hospital admission and support those important to them through the bereavement process.

3. Initiate and support advance care planning, designing and embedding the documentation, culture and education required to sustain this process.

4. Provide EoLC education through the ABC programme, training the staff to use a supportive care register.

Evaluation and sustainability $\mathrm{The} \mathrm{ABC}$ end of life care education programme uses a Palliative and EoLC Checklist for care homes for each patient, to record all aspects of support in place, and measure improvements in care. This will be the outcome tool for the Dementia Nurse project. The project is co-funded by Hospice UK and St James Place; the Hospice of St Francis is committed to continuing the post at the project end.

\section{P-136 ADVANCE CARE PLANNING AND ADVANCE HEALTHCARE DIRECTIVES WITH A PERSON WITH DEMENTIA}

${ }^{1}$ Deirdre Shanagher, ${ }^{1}$ Marie Lynch, ${ }^{1} J o h n$ Weafer, ${ }^{2}$ Willie Molloy, ${ }^{3}$ Ruth Esther Beck, ${ }^{1}$ Patrica Rickard-Clarke, ${ }^{4}$ Sharon Beatty, ${ }^{5}$ Emer Begley. ${ }^{1}$ Irish Hospice Foundation, Dublin, Ireland; ${ }^{2}$ University College Cork and university Hospital Cork; ${ }^{3}$ Ulster University; ${ }^{4}$ University College Hospital Galway; ${ }^{5}$ The Alzheimer Society of Ireland
Background Dementia is a progressive life-limiting illness. People with dementia value planning ahead. It allows them to express wishes and preferences and reduces anxiety. With the enactment of The Assisted Decision Making (Capacity) Act 2015 guidance in relation to advance care planning and advance healthcare directives with people with dementia is required by health and social care professionals.

Methods An expert advisory group was established. A systematic literature review, searching online databases, CINAHL and PubMed was carried out. Grey literature was also accessed. The themes were presided on by the expert advisory group. Identified literature review themes directed the scope of the guidance.

Results 288 articles were deemed appropriate. Post review with the expert advisory group the scope of the guidance document was extended to reach the person with dementia and family carers as well as healthcare staff across all settings.

The themes from the literature include:

1. Advance care planning and advance healthcare directives with people with dementia

a. Advance care planning is difficult to engage in due to fluctuating capacity.

2. Family members:

a. Uncertain about roles in advance care planning and having conversations.

3. Professional uncertainty

a. Time constraints, lack of knowledge and understanding of dementia, advance care planning and legal responsibilities are factors.

Guidance is offered on each on each of the above areas.

Conclusion A guidance document has been prepared for health and social care staff to provide palliative care to people with dementia. The document will be published and made available via the Irish Hospice Foundation website.

\section{P-137 SUPPORTING PEOPLE WITH DEMENTIA TO DIE AT HOME IN IRELAND}

Deirdre Shanagher, Sarah Cronin, Marie Lynch. Irish Hospice Foundation, Dublin, Ireland

10.1136/bmjspcare-2016-001245.160

Background The Irish Hospice Foundation has funded a nightnursing service for people with conditions other than cancer since 2006. The number of people with dementia referred to the service has risen each year since then. The Irish Hospice Foundation carried out an audit of all of the referrals received where people had a diagnosis of dementia between June and December 2015.

Aim The aim was to explore components of care which support a person with dementia who accessed the night nursing service to die at home.

Method Supplementary information from specialist palliative care teams (SPCT) was gathered for 52 dementia referrals to the night-nursing service between May and December 2015.

Results

- The availability of family/friends to provide care to a person with dementia appears to be a key determinant to them staying at home

- Having a supportive GP was important for a large number of the sample.

- The decision to stay at home was made by the person with dementia or their families in over $50 \%$ of the sample 